\title{
The Godunov method for a 2-phase model
}

\author{
Mauro Garavello ${ }^{*}$, Francesca Marcellini ${ }^{1}$ \\ ${ }^{1}$ Dipartimento di Matematica e Applicazioni, Università di Milano Bicocca, \\ Milano, Italy \\ *Email address for correspondence: mauro.garavello@unimib.it \\ Communicated by Roberto Natalini \\ Received on 12 19, 2016. Accepted on 06 16, 2017.
}

\begin{abstract}
We consider the Godunov numerical method to the phase-transition traffic model, proposed in [1], by Colombo, Marcellini, and Rascle. Numerical tests are shown to prove the validity of the method. Moreover we highlight the differences between such model and the one proposed in [2], by Blandin, Work, Goatin, Piccoli, and Bayen.

Keywords: 2--Phase Model, Continuum Traffic Models, Godunov Scheme, Hyperbolic Systems of Conservation Laws.

AMS subject classification: 35L65, 90B20
\end{abstract}

\section{Introduction}

Aim of this paper is to present the Godunov method for approximating the solutions to the 2-phase traffic model, introduced in [1] by Colombo, Marcellini, and Rascle. The model consists on the system of conservation laws

(1) $\left\{\begin{array}{l}\partial_{t} \rho+\partial_{x}(\rho v(\rho, \eta))=0 \\ \partial_{t} \eta+\partial_{x}(\eta v(\rho, \eta))=0\end{array} \quad\right.$ with $\quad v(\rho, \eta)=\min \left\{V_{\max }, \frac{\eta}{\rho} \psi(\rho)\right\}$,

where $\rho=\rho(t, x)$ is the car density at time $t>0$ and at position $x \in \mathbb{R}$, $\eta=\eta(t, x)$ is a generalized momentum, $v=v(\rho, \eta)$ is the average speed of cars, $V_{\max }$ is a positive constant describing the maximum speed of cars, and $\psi=\psi(\rho)$ is a given decreasing function. This model is an extension of the famous Lighthill-Whitham-Richards (LWR) model, see [3,4], and it is obtained by assuming that different kinds of drivers have different maximal speeds $w=w(\rho, \eta)$, where $w=\eta / \rho \in[\check{w}, \hat{w}]$, for suitable constants $0<\check{w}<\hat{w}$.

The model described by (1) belongs to the class of hyperbolic phase transition models for traffic, whose aim is to describe the different traffic 


\section{Garavello, F. Marcellini}

regimes; see [5]. The pioneer work in this class is the model proposed in 2002 by Colombo; see [6-8]. Its distinctive feature is that the two phases are disconnected. Subsequently other phase transition models have been introduced in the literature; see [1,2,9-11]. In particular, the models in [1,2], although very similar in the fundamental diagram, are indeed different, since solutions to Riemann problems have different structures and contain a different number of waves. Also the derivation of the two models is completely different: the construction in [2] is done imposing a priori two phases, the free and the congested one, while in [1] the two phases are obtained as a consequence of the speed limit $V_{\max }$.

In the present paper, we describe the Godunov numerical scheme for system (1). The Godunov method is a finite volume method for conservation laws; it is based on the solution to the Riemann problem in order to approximate the flux between two contiguous cells; see [12,13]. In our setting, the fundamental diagram in the conserved variable is convex; hence the application of this method is straightforward. Instead the nonconvexity of the fundamental diagram for the model in [2] is a source of various problems for the Godunov method; see [14,15].

The paper is organized as follow. In Section 2 we describe the 2-Phase Traffic Model (1). In Section 3 we make a comparison between the 2-Phase model (1) and that in [2]. Finally in Section 4, we describe the Godunov method to the model (1). Some numerical integrations conclude the paper.

\section{Notations and Description of the Phase Transition Model}

In this section we fix notations and we recall some properties concerning the 2-Phase traffic model introduced in [1]. As already said, the model (1) is an extension of the classical LWR model, given by the following scalar conservation law

$$
\partial_{t} \rho+\partial_{x}(\rho V)=0,
$$

where $\rho$ is the traffic density and $V=V(t, x, \rho)$ is the speed.

We consider the following two assumptions on the speed:

- We assume that, at a given density, different drivers may choose different velocities, that is, we assume that $V=w \psi(\rho)$, where $\psi=$ $\psi(\rho)$ is a $\mathbf{C}^{2}$ function and $w=w(t, x)$ is the maximal speed of a driver, located at position $x$ at time $t$.

- We impose an overall bound on the speed $V_{\max }$. 


\section{The Godunov method for a 2-phase model}


Figure 1. The free phase $F$ and the congested phase $C$ resulting from (1) in the coordinates, from left to right, $(\rho, \eta)$ and $(\rho, \rho v)$.

We get the following $2 \times 2$ system

$$
\left\{\begin{array}{l}
\partial_{t} \rho+\partial_{x}(\rho v)=0 \\
\partial_{t} w+v \partial_{x} w=0
\end{array} \quad \text { with } \quad v=\min \left\{V_{\max }, w \psi(\rho)\right\}\right.
$$

and then the model in (1), with the change of variables $\eta=\rho w$.

As a consequence of the introduction of the speed bound $V_{\max }$, system (1) produces two phases, the free and the congested one, described by the sets

$$
\begin{aligned}
& F=\left\{(\rho, w) \in[0, R] \times[\check{w}, \hat{w}]: v(\rho, \rho w)=V_{\max }\right\}, \\
& C=\{(\rho, w) \in[0, R] \times[\check{w}, \hat{w}]: v(\rho, \rho w)=w \psi(\rho)\} .
\end{aligned}
$$

In Figure 1 it is represented the fundamental diagram in the coordinates $(\rho, \eta)$ and $(\rho, \rho v)$. Note that $F$ and $C$ are closed sets and $F \cap C \neq \emptyset$. Note also that $F$ is one-dimensional in the $(\rho, \rho v)$ plane of the fundamental diagram, while it is two-dimensional in the $(\rho, \eta)$ coordinates.

We recall the following assumptions:

(H-1) $R, \check{w}, \hat{w}, V_{\max }$ are positive constants, with $V_{\max }<\check{w}<\hat{w}$.

(H-2) $\psi \in \mathbf{C}^{\mathbf{2}}([0, R] ;[0,1])$ is such that

$$
\begin{aligned}
\psi(0) & =1, & \psi(R) & =0, \\
\psi^{\prime}(\rho) & \leq 0, & \frac{d^{2}}{d \rho^{2}}(\rho \psi(\rho)) & \leq 0 \quad \text { for all } \rho \in[0, R] .
\end{aligned}
$$

(H-3) Waves of the first family in the congested phase $C$ have negative speed.

We recall the eigenvalues, right eigenvectors, and Lax curves $\eta=$ 


\section{Garavello, F. Marcellini}

$\mathcal{L}_{i}\left(\rho ; \rho_{o}, \eta_{o}\right)$ in $C:$

$$
\begin{aligned}
\lambda_{1}(\rho, \eta) & =\eta \psi^{\prime}(\rho)+v(\rho, \eta), & \lambda_{2}(\rho, \eta) & =v(\rho, \eta), \\
r_{1}(\rho, \eta) & =\left[\begin{array}{c}
-\rho \\
-\eta
\end{array}\right], & r_{2}(\rho, \eta) & =\left[\begin{array}{c}
1 \\
\eta\left(\frac{1}{\rho}-\frac{\psi^{\prime}(\rho)}{\psi(\rho)}\right)
\end{array}\right], \\
\nabla \lambda_{1} \cdot r_{1} & =-\frac{d^{2}}{d \rho^{2}}[\rho \psi(\rho)], & \nabla \lambda_{2} \cdot r_{2} & =0, \\
\mathcal{L}_{1}\left(\rho ; \rho_{o}, \eta_{o}\right) & =\eta_{o} \frac{\rho}{\rho_{o}}, & \mathcal{L}_{2}\left(\rho ; \rho_{o}, \eta_{o}\right) & =\frac{\rho v\left(\rho_{o}, \eta_{o}\right)}{\psi(\rho)}, \rho_{o}<R .
\end{aligned}
$$

When $\rho_{o}=R$, the 2-Lax curve through $\left(\rho_{o}, \eta_{o}\right)$ is the segment $\rho=R$, $\eta \in[R \check{w}, R \hat{w}]$.

In order to apply the Godunov's method in Section 4, we recall the description of the solutions of the Riemann problem for the model (1). First, we recall all the possible waves in the solution.

- A Linear wave is a wave connecting two states in the free phase.

- A Phase Transition Wave is a wave connecting a left state $\left(\rho_{l}, \eta_{l}\right) \in$ $F$ with a right state $\left(\rho_{r}, \eta_{r}\right) \in C$ satisfying $\frac{\eta_{l}}{\rho_{l}}=\frac{\eta_{r}}{\rho_{r}}$.

- A Wave of the First Family is a wave connecting a left state $\left(\rho_{l}, \eta_{l}\right) \in$ $C$ with a right state $\left(\rho_{r}, \eta_{r}\right) \in C$ such that $\frac{\eta_{l}}{\rho_{l}}=\frac{\eta_{r}}{\rho_{r}}$.

- A Wave of the Second Family is a wave connecting a left state $\left(\rho_{l}, \eta_{l}\right) \in C$ with a right state $\left(\rho_{r}, \eta_{r}\right) \in C$ such that $v\left(\rho_{l}, \eta_{l}\right)=$ $v\left(\rho_{r}, \eta_{r}\right)$.

\subsection{The Riemann Problem}

In this subsection we describe the Riemann problem for the system (1), see also [1].

Under the assumptions $(\mathbf{H}-\mathbf{1}),(\mathbf{H}-2)$ and $(\mathbf{H}-3)$, for all states $\left(\rho^{l}, \eta^{l}\right)$ and $\left(\rho^{r}, \eta^{r}\right) \in F \cup C$, the Riemann problem consisting of (1) with initial data

$$
\rho(0, x)=\left\{\begin{array}{ll}
\rho^{l} & \text { if } x<0 \\
\rho^{r} & \text { if } x>0
\end{array} \quad \eta(0, x)= \begin{cases}\eta^{l} & \text { if } x<0 \\
\eta^{r} & \text { if } x>0\end{cases}\right.
$$

admits a unique self similar weak solution $(\rho, \eta)=(\rho, \eta)(t, x)$ constructed as follows:

1. If $\left(\rho^{l}, \eta^{l}\right),\left(\rho^{r}, \eta^{r}\right) \in F$, then system (1) reduces to the degenerate linear system

$$
\left\{\begin{array}{l}
\partial_{t} \rho+\partial_{x}\left(\rho V_{\max }\right)=0 \\
\partial_{t} \eta+\partial_{x}\left(\eta V_{\max }\right)=0
\end{array}\right.
$$




\section{The Godunov method for a 2-phase model}
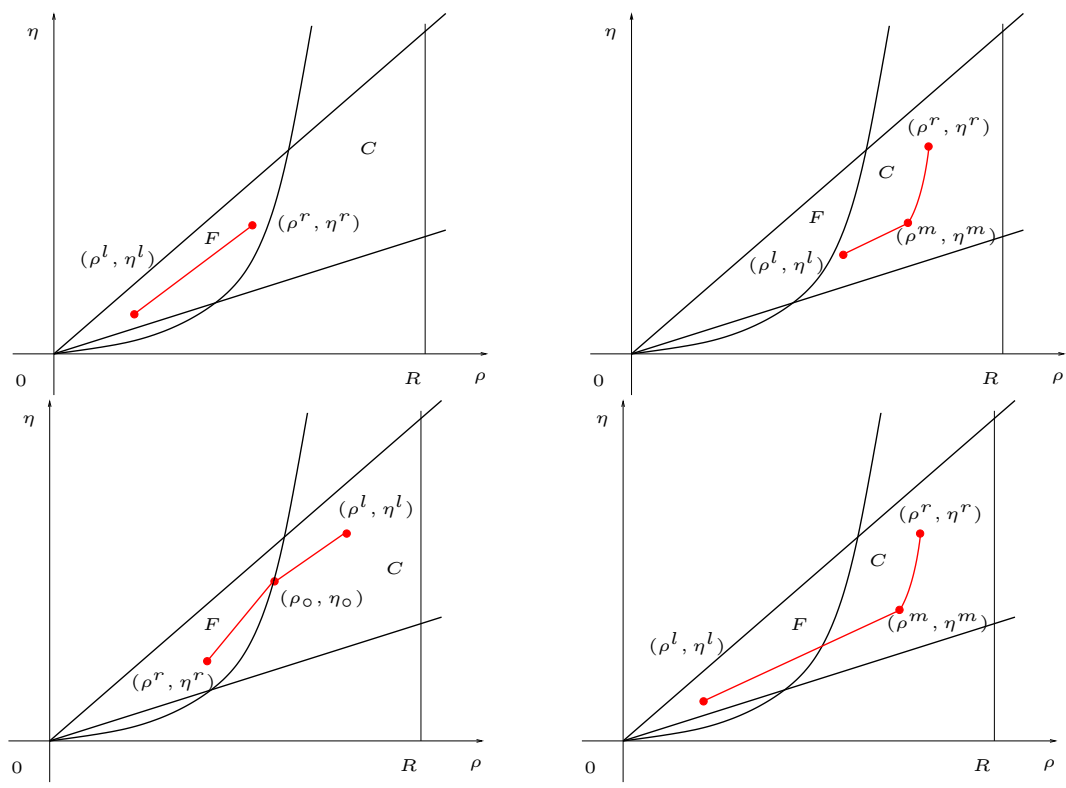

Figure 2. Above, the Riemann problem for (1) if $\left(\rho^{l}, \eta^{l}\right),\left(\rho^{r}, \eta^{r}\right) \in F$, left, if $\left(\rho^{l}, \eta^{l}\right),\left(\rho^{r}, \eta^{r}\right) \in C$, right. Below, the Riemann problem for $(1)$ if $\left(\rho^{l}, \eta^{l}\right) \in C$ and $\left(\rho^{r}, \eta^{r}\right) \in F$, left, if $\left(\rho^{l}, \eta^{l}\right) \in F$ and $\left(\rho^{r}, \eta^{r}\right) \in C$, right.

The solution attains values in $F$ and consists of a linear wave separating $\left(\rho^{l}, \eta^{l}\right)$ from $\left(\rho^{r}, \eta^{r}\right)$, see Figure 13, above, left.

2. If $\left(\rho^{l}, \eta^{l}\right),\left(\rho^{r}, \eta^{r}\right) \in C$, then system (1) is given by

$$
\left\{\begin{array}{l}
\partial_{t} \rho+\partial_{x}(\eta \psi(\rho))=0 \\
\partial_{t} \eta+\partial_{x}\left(\frac{\eta^{2}}{\rho} \psi(\rho)\right)=0 .
\end{array}\right.
$$

The solution attains values in $C$ and consists of a wave of the first family (shock or rarefaction) between $\left(\rho^{l}, \eta^{l}\right)$ and a middle state $\left(\rho^{m}, \eta^{m}\right)$, followed by a wave of the second family between $\left(\rho^{m}, \eta^{m}\right)$ and $\left(\rho^{r}, \eta^{r}\right)$. The middle state $\left(\rho^{m}, \eta^{m}\right)$ is in $C$ and is uniquely characterized by the two conditions $\frac{\eta^{m}}{\rho^{m}}=\frac{\eta^{l}}{\rho^{l}}$ and $v\left(\rho^{m}, \eta^{m}\right)=v\left(\rho^{r}, \eta^{r}\right)$, see Figure 13, above, right.

3. If $\left(\rho^{l}, \eta^{l}\right) \in C$ and $\left(\rho^{r}, \eta^{r}\right) \in F$, then the solution attains values in $F \cup C$ and consists of a wave of the first family separating $\left(\rho^{l}, \eta^{l}\right)$ from a middle state $\left(\rho_{\circ}, \eta_{\circ}\right)$ and by a linear wave separating $\left(\rho_{\circ}, \eta_{\circ}\right)$ from $\left(\rho^{r}, \eta^{r}\right)$. The middle state $\left(\rho_{\circ}, \eta_{\circ}\right)$ belongs to the intersection between $F$ and $C$ and is uniquely characterized by the two conditions $\frac{\eta_{\circ}}{\rho_{\circ}}=\frac{\eta^{r}}{\rho^{r}}$ and $v\left(\rho_{\circ}, \eta_{\circ}\right)=V_{\max }$, see Figure 13, below, left.

4. If $\left(\rho^{l}, \eta^{l}\right) \in F$ and $\left(\rho^{r}, \eta^{r}\right) \in C$, then the solution attains values in $F \cup C$ and consists of a phase transition wave between $\left(\rho^{l}, \eta^{l}\right)$ and a 


\section{Garavello, F. Marcellini}

middle state $\left(\rho^{m}, \eta^{m}\right)$, followed by a wave of the second family between $\left(\rho^{m}, \eta^{m}\right)$ and $\left(\rho^{r}, \eta^{r}\right)$. The middle state $\left(\rho^{m}, \eta^{m}\right)$ is in $C$ and is uniquely characterized by the two conditions $\frac{\eta^{m}}{\rho^{m}}=\frac{\eta^{l}}{\rho^{l}}$ and $v\left(\rho^{m}, \eta^{m}\right)=v\left(\rho^{r}, \eta^{r}\right)$, see Figure 13, below, right.

\section{Comparison with the Model in [2]}

In this section we briefly describe the 2-Phase traffic model, introduced by Blandin, Work, Goatin, Piccoli and Bayen in [2], and we show the main differences with (1).

The phase transition traffic model in [2] has been developed as an extension of the Colombo phase transition model [6] and can be described by the following system

$$
\begin{cases}\partial_{t} \rho+\partial_{x}(\rho V)=0, & \text { if }(\rho, q) \in \Omega_{f}, \\
\left\{\begin{array}{l}
\partial_{t} \rho+\partial_{x}(\rho v(\rho, q))=0, \\
\partial_{t} q+\partial_{x}(q v(\rho, q))=0 .
\end{array}\right. & \text { if }(\rho, q) \in \Omega_{c}\end{cases}
$$

where $\rho$ and $q$ represent, respectively, the car traffic density and the linearized momentum, $v=v(\rho, q)$ is the average speed of cars, while the sets $\Omega_{f}$ and $\Omega_{c}$, respectively the free and the congested phases, are given by

$$
\begin{aligned}
& \Omega_{f}=\left\{(\rho, q) \in[0, R] \times\left[0,+\infty\left[: q=\frac{R(\rho-\sigma)}{\sigma(R-\rho)}, 0 \leq \rho \leq \sigma_{+}\right\}\right.\right. \\
& \Omega_{c}=\left\{(\rho, q) \in[0, R] \times\left[0,+\infty\left[: v(\rho, q) \leq V, \frac{q^{-}}{R} \leq \frac{q}{\rho} \leq \frac{q^{+}}{R}\right\} .\right.\right.
\end{aligned}
$$

In (7), $R>0$ denotes the maximal density, $V>0$ is the maximal velocity attained by the vehicles in the free phase, $q^{-}$and $q^{+}$are respectively the minimum and the maximum value of the momentum $q$, and the constants $\sigma$ and $\sigma_{+}$are suitable constants in $[0, R]$. The velocity $v: \Omega_{f} \cup \Omega_{c} \rightarrow[0,+\infty[$ is defined by

$$
\text { (8) } v(\rho, q)= \begin{cases}V, & \text { if }(\rho, q) \in \Omega_{f} \\ v^{e q}(\rho)(1+q)=\frac{V \sigma}{R-\sigma}\left(\frac{R}{\rho}-1\right)(1+q), & \text { if }(\rho, q) \in \Omega_{c}\end{cases}
$$

where the equilibrium velocity $v^{e q}$ represents the desired speed of cars when the density is $\rho$. In the congested phase $\Omega_{c}$, when $q=0$, the velocity of cars is the equilibrium velocity $v^{e q}$.

The models (1) and (6) have some similarities. Both models are described by two intersecting phases: the free phase and the two-dimensional congested phase. In Figures 1 and 3 the fundamental diagrams, respectively for (1) and for (6), are drawn. Note however that the free phase in (6) is 

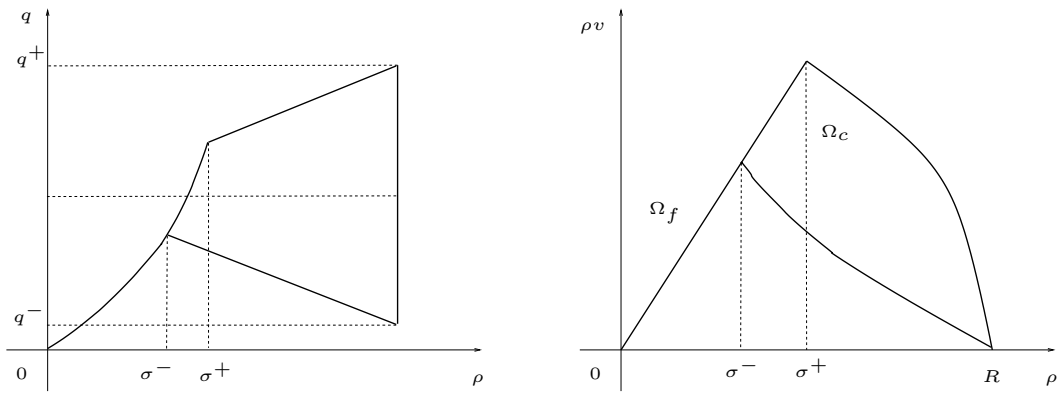

Figure 3. The fundamental diagram in [2] in the coordinates, from left to right, $(\rho, q)$ and $(\rho, \rho v)$. Note that free phase $\Omega_{f}$ is one-dimensional, while the congested phase $\Omega_{c}$ is two-dimensional.

one-dimensional both in the conserved quantity coordinates $(\rho, q)$ and in the coordinates $(\rho, \rho v)$. In the model (1) the free phase is two-dimensional in the conserved quantity coordinates $(\rho, \eta)$.

The main difference between (1) and (6) lies in the solution of the Riemann problem, in particular when the left state belongs to the free phase and the right state to the congested one.

The following result for (1) holds.

Proposition 3.1. Consider the system (1) and fix the states $\left(\rho^{l}, \eta^{l}\right) \in F$, $\left(\rho^{r}, \eta^{r}\right) \in C$. The Riemann problem for (1) with the initial condition

$$
\left(\rho_{0}, \eta_{0}\right)(x)= \begin{cases}\left(\rho^{l}, \eta^{l}\right) & \text { if } x<0 \\ \left(\rho^{r}, \eta^{r}\right) & \text { if } x>0\end{cases}
$$

is solved with at most two waves.

Proof. Since $\left(\rho^{l}, \eta^{l}\right) \in F$ and $\left(\rho^{r}, \eta^{r}\right) \in C$, the solution to the Riemann problem consists of a shock wave connecting $\left(\rho^{l}, \eta^{l}\right)$ to a middle state $\left(\rho^{m}, \eta^{m}\right)$, possibly followed by a wave of the second family connecting $\left(\rho^{m}, \eta^{m}\right)$ to $\left(\rho^{r}, \eta^{r}\right)$; see Subsection 2.1 and Figure 4. This concludes the proof.

The following result for (6) holds.

Proposition 3.2. Consider the system (6) and fix the states $\left(\rho^{l}, q^{l}\right) \in \Omega_{f}$, $\left(\rho^{r}, q^{r}\right) \in \Omega_{c}$. The Riemann problem for (6) with the initial condition

$$
\left(\rho_{0}, q_{0}\right)(x)= \begin{cases}\left(\rho^{l}, q^{l}\right) & \text { if } x<0 \\ \left(\rho^{r}, q^{r}\right) & \text { if } x>0\end{cases}
$$

is solved with at most three waves. 


\section{Garavello, F. Marcellini}

Proof. We use here the notations in [2]. First define the intermediate state $\left(\rho^{m}, q^{m}\right) \in \Omega_{c}$ as the solution to the system

$$
\left\{\begin{array}{l}
\frac{q^{m}}{\rho^{m}}=\frac{q}{R} \\
v_{c}\left(\rho^{m}, q^{m}\right)=v_{c}\left(\rho^{r}, q^{r}\right) .
\end{array}\right.
$$

Let $\Lambda\left(\left(\rho^{l}, q^{l}\right),\left(\rho^{m}, q^{m}\right)\right)$ be the speed of the phase transition wave connecting $\left(\rho^{l}, q^{l}\right)$ to $\left(\rho^{m}, q^{m}\right)$. Its value is given by the Rankine-Hugoniot condition

$$
\Lambda\left(\left(\rho^{l}, q^{l}\right),\left(\rho^{m}, q^{m}\right)\right)=\frac{\rho^{m} v\left(\rho^{m}, q^{m}\right)-\rho^{l} V}{\rho^{m}-\rho^{l}} .
$$

Denoting with $\lambda_{1}\left(\rho^{m}, q^{m}\right)$ the first eigenvalue of the Jacobian matrix of the flux at $\left(\rho^{m}, q^{m}\right)$, the following possibilities hold.

1. $\Lambda\left(\left(\rho^{l}, q^{l}\right),\left(\rho^{m}, q^{m}\right)\right) \geq \lambda_{1}\left(\rho^{m}, q^{m}\right)$. In this case, the solution to the Riemann problem consists of a phase transition wave connecting $\left(\rho^{l}, q^{l}\right)$ to $\left(\rho^{m}, q^{m}\right)$, possibly followed by a contact discontinuity wave connecting $\left(\rho^{m}, q^{m}\right)$ to $\left(\rho^{r}, q^{r}\right)$; see Figure 4 .

2. $\Lambda\left(\left(\rho^{l}, q^{l}\right),\left(\rho^{m}, q^{m}\right)\right)<\lambda_{1}\left(\rho^{m}, q^{m}\right)$. Define $\left(\rho^{p}, q^{p}\right) \in \Omega_{c}$ the solution to

$$
\left\{\begin{array}{l}
\frac{q^{p}}{\rho^{p}}=\frac{q^{-}}{R} \\
\Lambda\left(\left(\rho^{l}, q^{l}\right),\left(\rho^{p}, q^{p}\right)\right)=\lambda_{1}\left(\rho^{p}, q^{p}\right) .
\end{array}\right.
$$

The solution to the Riemann problem consists of a phase transition wave connecting $\left(\rho^{l}, q^{l}\right)$ to $\left(\rho^{p}, q^{p}\right)$, of a rarefaction wave of the first family connecting $\left(\rho^{p}, q^{p}\right)$ to $\left(\rho^{m}, q^{m}\right)$, and of contact discontinuity wave connecting $\left(\rho^{m}, q^{m}\right)$ to $\left(\rho^{r}, q^{r}\right)$; see Figure 4 .

The proof is so concluded.

\section{The Godunov Method}

In this section we describe the Godunov method for the model (1) under the assumptions $\mathbf{( H - 1 )}, \mathbf{( H - 2 )}$, and $\mathbf{( H - 3 )}$; see $[12,13,16]$. We recall that such numerical scheme is a finite volume method, based on Riemann problems within computational cells in order to obtain the numerical fluxes.

Consider the system (1), with the conserved variables $(\rho, \eta)$; see Figure 1. Introduce the space discretization $\Delta x$ and the time discretization $\Delta t$ satisfying the Courant-Friedrichs-Lewy conditions (see [17]) as in [16]. Define $\nu=\Delta t / \Delta x$, and, for all $j \in \mathbb{Z}$ and all $n \in \mathbb{N}$, the points $x_{j+1 / 2}=j \Delta x, x_{j}=(j-1 / 2) \Delta x$, the time $t^{n}=n \Delta t$, and the cell $C_{j}^{n}=\left\{t^{n}\right\} \times\left[x_{j-1 / 2}, x_{j+1 / 2}\right)$ of length $\Delta x$.

The Godunov method consists in the following steps. 


\section{The Godunov method for a 2-phase model}
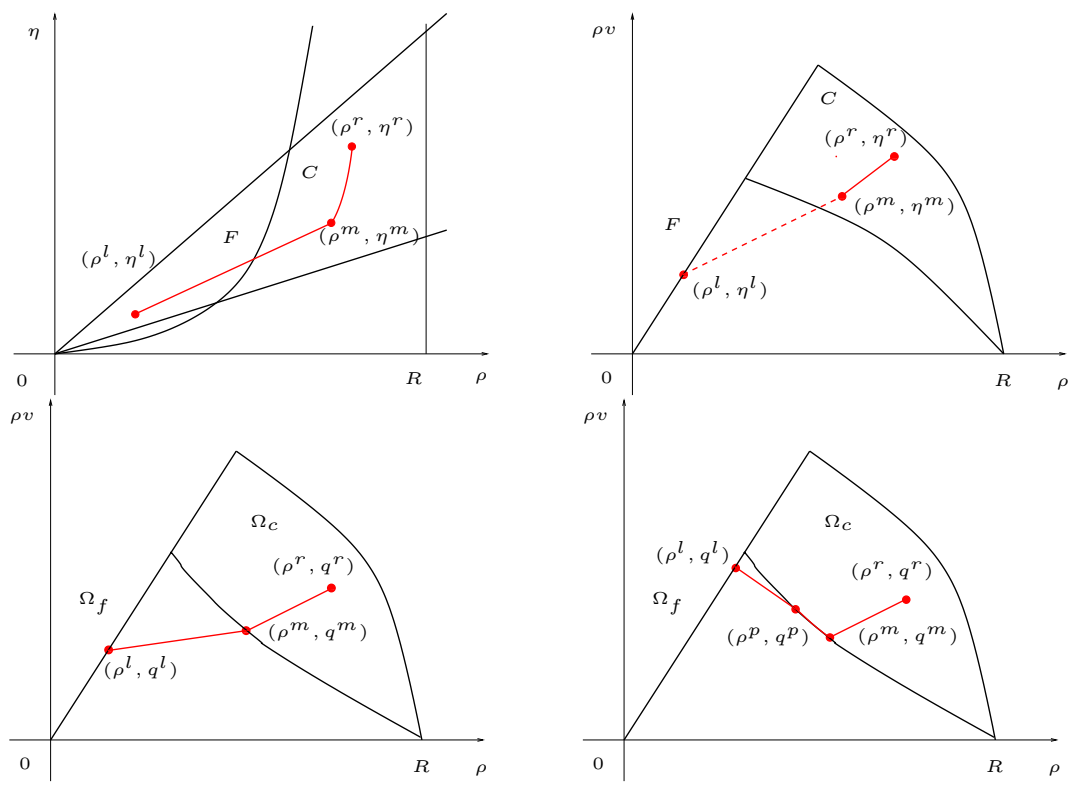

Figure 4. Above, the Riemann problem for $(1)$ if $\left(\rho^{l}, \eta^{l}\right) \in F$ and $\left(\rho^{r}, \eta^{r}\right) \in C$, left, in the $(\rho, \eta)$ coordinates, right, in the $(\rho, \rho v)$ coordinates. Below, the Riemann problem for (6) if $\left(\rho^{l}, q^{l}\right) \in \Omega_{f}$ and $\left(\rho^{r}, q^{r}\right) \in \Omega_{c}$, left, the first case in the proof of Proposition 3.2, right the second case in the proof of Proposition 3.2.

1. Approximate an initial condition $\left(\rho_{0}, \eta_{0}\right)$ by a function $\left(\rho_{\nu}(0, \cdot), \eta_{\nu}(0, \cdot)\right)$, constant in each cell $C_{j}^{0}$. Define $u_{j}^{0}=\left(\rho_{j}^{0}, \eta_{j}^{0}\right)=\left(\rho_{\nu}\left(0, x_{j}\right), \eta_{\nu}\left(0, x_{j}\right)\right)$ for every $j \in \mathbb{Z}$.

2. Construct, for every $n \in \mathbb{N}$ and $j \in \mathbb{Z}$, the values $u_{j}^{n+1}$ by the recurrence formula

$$
\begin{aligned}
u_{j}^{n+1} & =\left(\rho_{j}^{n+1}, \eta_{j}^{n+1}\right)=u_{j}^{n}-\frac{\Delta t}{\Delta x}\left(\mathcal{F}_{j+1 / 2}^{n}-\mathcal{F}_{j-1 / 2}^{n}\right) \\
& =\left(\rho_{j}^{n}, \eta_{j}^{n}\right)-\frac{\Delta t}{\Delta x}\left[\left(F_{j+1 / 2}^{n}, G_{j+1 / 2}^{n}\right)-\left(F_{j-1 / 2}^{n}, G_{j-1 / 2}^{n}\right)\right],
\end{aligned}
$$

where $\mathcal{F}_{j+1 / 2}^{n}=\left(F_{j+1 / 2}^{n}, G_{j+1 / 2}^{n}\right)$ is a numerical flux at the interface $x_{j+1 / 2}$, obtained through the solution of corresponding Riemann problems.

If $j \in \mathbb{Z}$, the numerical flux $\mathcal{F}_{j+1 / 2}^{n}=\left(F_{j+1 / 2}^{n}, G_{j+1 / 2}^{n}\right)$ depends on the values $u_{j}^{n}$ and $u_{j+1}^{n}$. Namely, denoting for simplicity with $u_{l}=\left(\rho_{l}, \eta_{l}\right)$ and 


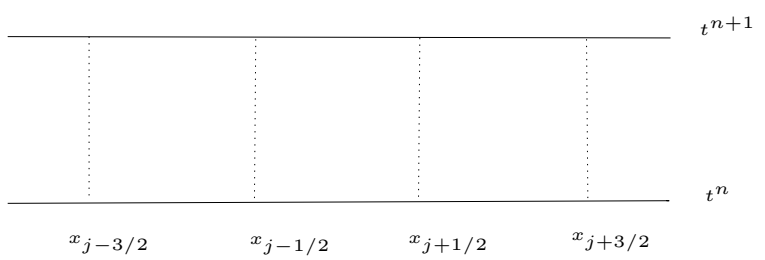

Figure 5. A cell given by $\left[x_{j-1 / 2}, x_{j+1 / 2}\right]$ in the Godunov Scheme.

$u_{r}=\left(\rho_{r}, \eta_{r}\right)$ respectively $u_{j}^{n}$ and $u_{j+1}^{n}$, we have

$$
F_{j+1 / 2}^{n}=\left\{\begin{array}{lll}
\rho_{l} V_{\max } & \text { if } & u_{l}, u_{r} \in F \\
\eta_{m} \psi\left(\rho_{m}\right) & \text { if } & u_{l}, u_{r} \in C \\
\rho_{\circ} V_{\max } & \text { if } & u_{l} \in C, u_{r} \in F \\
\eta_{m} \psi\left(\rho_{m}\right) & \text { if } & u_{l} \in F, u_{r} \in C, \rho_{l} V_{\max }>\eta_{m} \psi\left(\rho_{m}\right) \\
\rho_{l} V_{\max } & \text { if } & u_{l} \in F, u_{r} \in C, \rho_{l} V_{\max }<\eta_{m} \psi\left(\rho_{m}\right)
\end{array}\right.
$$

and

$$
G_{j+1 / 2}^{n}=\left\{\begin{array}{lll}
\eta_{l} V_{\max } & \text { if } & u_{l}, u_{r} \in F \\
\frac{\eta_{m}^{2}}{\rho_{m}} \psi\left(\rho_{m}\right) & \text { if } & u_{l}, u_{r} \in C \\
\eta_{\circ} V_{\max } & \text { if } & u_{l} \in C, u_{r} \in F \\
\frac{\eta_{m}^{2}}{\rho_{m}} \psi\left(\rho_{m}\right) & \text { if } & u_{l} \in F, u_{r} \in C, \rho_{l} V_{\max }>\eta_{m} \psi\left(\rho_{m}\right) \\
\eta_{l} V_{\max } & \text { if } & u_{l} \in F, u_{r} \in C, \rho_{l} V_{\max }<\eta_{m} \psi\left(\rho_{m}\right) .
\end{array}\right.
$$

Above $\left(\rho_{m}, \eta_{m}\right) \in C$ is the middle state in the solution to the Riemann problem (5); see Subsection 2.1. Moreover the point $\left(\rho_{\circ}, \eta_{\circ}\right) \in F \cap C$ is the middle point in the solution to the Riemann problem (5) when $u_{l} \in C$ and $u_{r} \in F$; see point (3) of Subsection 2.1. Note that the condition $\rho_{l} V_{\max }$ respectively greater or less than $\eta_{m} \psi\left(\rho_{m}\right)$ means that, by the RankineHugoniot condition, the phase transition wave connecting $\left(\rho_{l}, \eta_{l}\right) \in F$ and $\left(\rho_{m}, \eta_{m}\right) \in C$ has strictly negative or positive speed.

\subsection{Example: the case of a traffic light with increasing $w$}

We present here a simple situation of a road with a traffic light, which turns green at the initial time $t=0$. At the beginning all the vehicles are stopped at the maximal density and $w$ is linearly increasing between $\check{w}$ and $\hat{w}$. We assume that the road is $3 \mathrm{~km}$ long, modeled by the real interval $(0,3000)$ and the traffic light is located at position $x=500$.

We choose, for the model (1), the parameters

$$
R=1, \quad V_{\max }=60 \mathrm{Km} / \mathrm{h}, \quad \check{w}=120 \mathrm{Km} / \mathrm{h}, \quad \hat{w}=140 \mathrm{Km} / \mathrm{h},
$$




\section{The Godunov method for a 2-phase model}

with $\psi(\rho)=1-\rho$ and initial conditions

$$
\rho_{0}(x)=\left\{\begin{array}{l}
1, x \leq 500 \\
0, x>500
\end{array} \quad w_{0}(x)= \begin{cases}\check{w}+\frac{\hat{w}-\breve{w}}{500} x, & x \leq 500 \\
0, & x>500 .\end{cases}\right.
$$

In Figure 6 we show the contour plot for the numerical solution, obtained with the Godunov scheme, using the following numerical parameters:

$$
T=300 \mathrm{~s}, \quad \Delta x=1 \mathrm{~m}, \quad \Delta t=0.4 \frac{\Delta x}{V_{\max }} .
$$

Finally, Figure 7 displays the fundamental diagrams, obtained by the simulation.
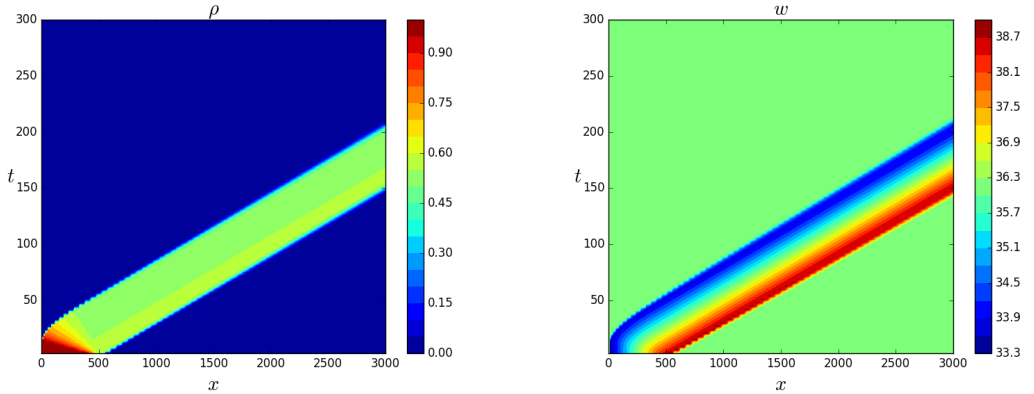

Figure 6. The contour plot for the density $\rho$ and for the maximal speed $w$ obtained with the Godunov method, with the parameters (12) and (14), and with $\psi(\rho)=1-\rho$. The initial condition are in (13).
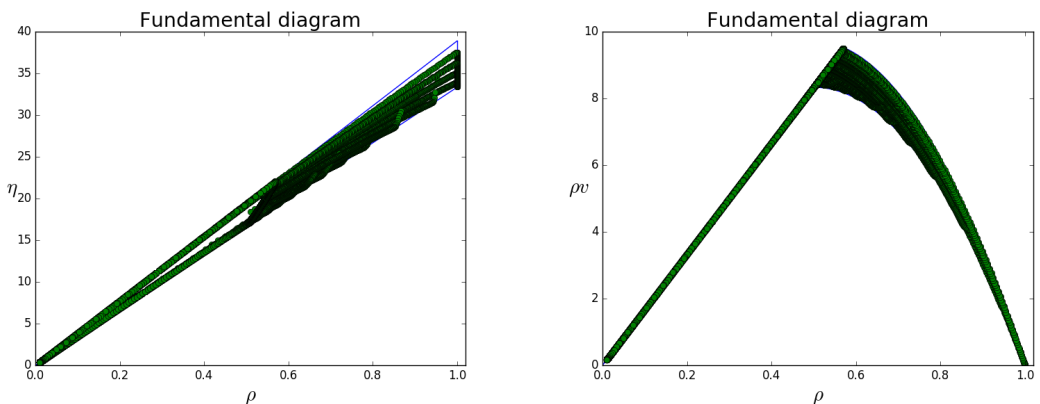

Figure 7. The fundamental diagrams in the planes $(\rho, \eta)$ and $(\rho, \rho v)$ obtained by the simulation of Subsection 4.1.

\subsection{Example: the case of a traffic light with decreasing $w$}

We consider here a situation very similar to that of Subsection 4.1. The only difference is that the initial $w$ is decreasing between $\check{w}$ and $\hat{w}$. As 
before, we assume that the road is $3 \mathrm{~km}$ long, modeled by the real interval $(0,3000)$ and the traffic light is located at position $x=500$.

We choose, for the model (1), the parameters (12), the function $\psi(\rho)=$ $1-\rho$, initial conditions

$$
\rho_{0}(x)=\left\{\begin{array}{ll}
1, x \leq 500 \\
0, x>500
\end{array} \quad w_{0}(x)= \begin{cases}\hat{w}+\frac{\check{w}-\hat{w}}{500} x, & x \leq 500 \\
0, & x>500\end{cases}\right.
$$

In Figure 8 we show the contour plot for the numerical solution, obtained with the Godunov scheme, using the numerical parameters (14). Finally, Figure 9 displays the fundamental diagrams, obtained by the simulation.
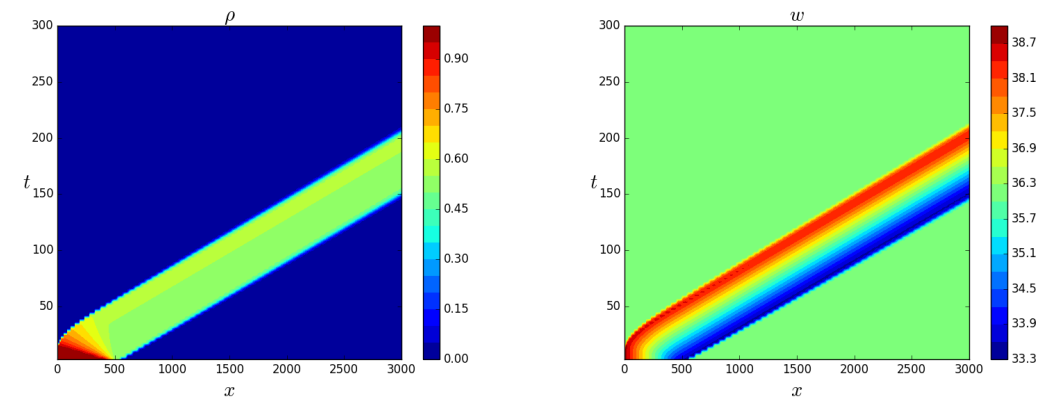

Figure 8. The contour plot for the density $\rho$ and for the maximal speed $w$ obtained with the Godunov method, with the parameters (12) and (14), and with $\psi(\rho)=1-\rho$. The initial condition are in (15).
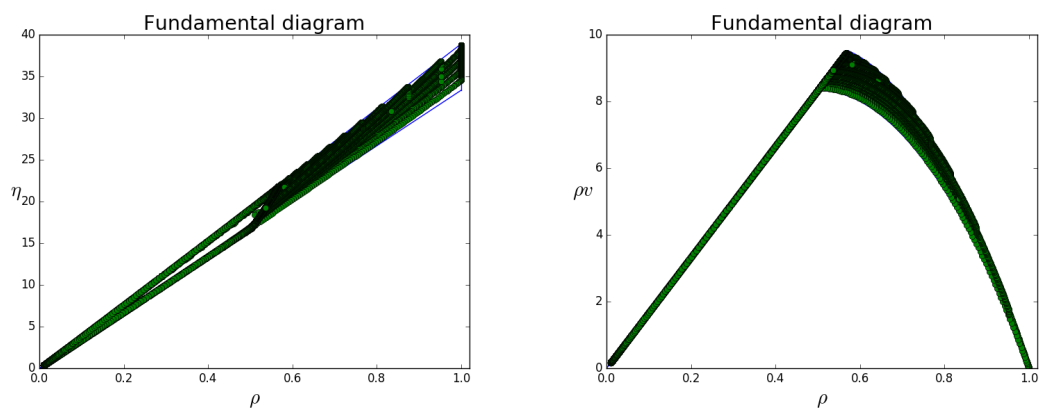

Figure 9. The fundamental diagrams in the planes $(\rho, \eta)$ and $(\rho, \rho v)$ obtained by the simulation of Subsection 4.2.

\subsection{Example: increasing $\rho$ and decreasing $w$}

In this part we consider an example where the initial conditions are monotone functions: the density $\rho$ is increasing, while the maximal speed 


\section{The Godunov method for a 2-phase model}

$w$ is decreasing. We assume that the road is $3 \mathrm{~km}$ long, modeled by the real interval $(0,3000)$.

We choose the parameters (12), the function $\psi(\rho)=1-\rho$, and the initial conditions

$$
\begin{aligned}
& \rho_{0}(x)= \begin{cases}0, & x \leq 500 \\
0.2+0.5 \frac{x-500}{2000}, & 500<x<2500 \\
0, & x>2500\end{cases} \\
& w_{0}(x)= \begin{cases}0, & x \leq 500 \\
\hat{w}+\frac{\check{w}-\hat{w}}{2000}(x-500) & , 500<x<2500 \\
0, & x \geq 2500 .\end{cases}
\end{aligned}
$$

In Figure 10 we show the contour plot for the numerical solution, obtained with the Godunov scheme, using the following numerical parameters:

$$
T=200 \mathrm{~s}, \quad \Delta x=1 \mathrm{~m}, \quad \Delta t=0.4 \frac{\Delta x}{V_{\max }} .
$$

Finally, Figure 11 displays the fundamental diagrams, obtained by the simulation.
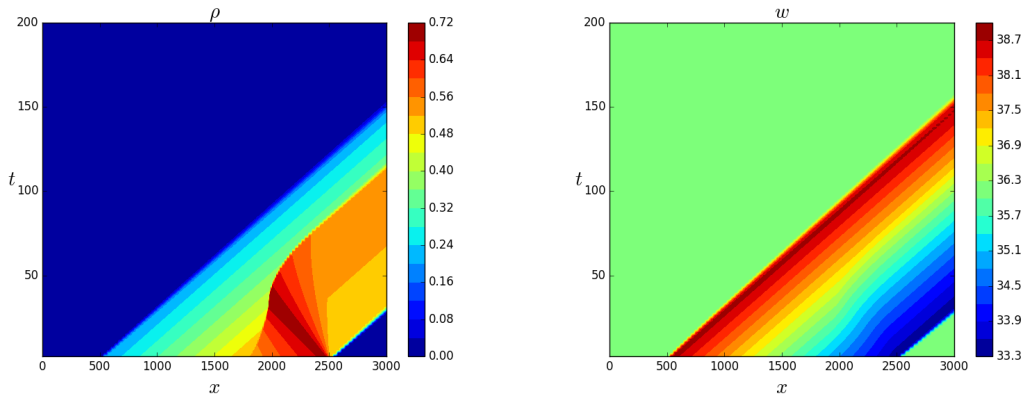

Figure 10. The contour plot for the density $\rho$ and for the maximal speed $w$ obtained with the Godunov method, with the parameters (12) and (17), and with $\psi(\rho)=1-\rho$. The initial condition are in (16). A shock curve is generated at about $x \sim 1800$ and interacts with a rarefaction curve generated at about $x \sim 2500$.

\subsection{Example: Interaction of two Riemann Problems}

In this part we consider an example where the initial conditions are piecewise constants with two points of discontinuity. The solution of this Cauchy problem can be obtained by solving two Riemann problems at the initial time and by solving additional Riemann problems when waves interact together. We assume that the road is $3 \mathrm{~km}$ long, modeled by the real interval $(0,3000)$. 


\section{Garavello, F. Marcellini}
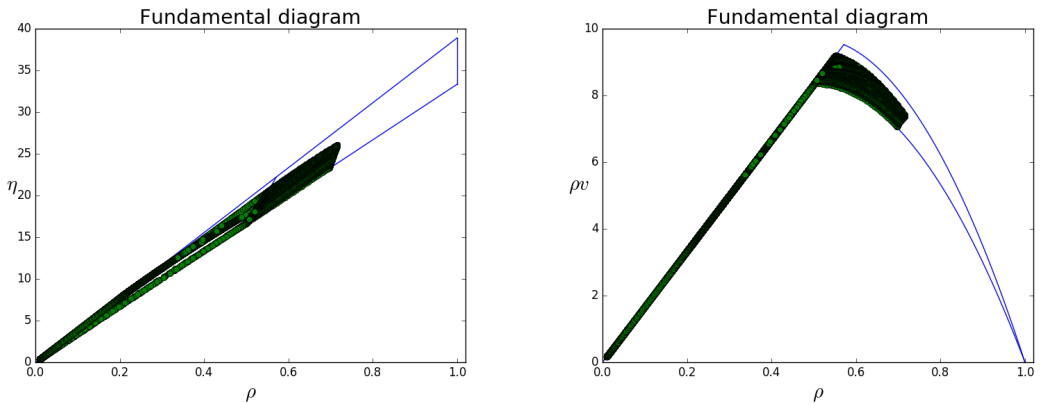

Figure 11. The fundamental diagrams in the planes $(\rho, \eta)$ and $(\rho, \rho v)$ obtained by the simulation of Subsection 4.3 .

We choose the parameters (12), the function $\psi(\rho)=1-\rho$, and the initial conditions

$$
\begin{aligned}
& \rho_{0}(x)=\left\{\begin{array}{l}
0.7, \quad x \leq 1000 \\
0.3,1000<x<2000 \\
0.92, x>2000
\end{array}\right. \\
& w_{0}(x)=\left\{\begin{array}{l}
38.33, x \leq 1000 \\
33.88,1000<x<2000 \\
36.11, x \geq 2000
\end{array}\right.
\end{aligned}
$$

In Figure 12 we show the contour plot for the numerical solution, obtained with the Godunov scheme, using the following numerical parameters:

$$
T=100 \mathrm{~s}, \quad \Delta x=1 \mathrm{~m}, \quad \Delta t=0.4 \frac{\Delta x}{V_{\max }} .
$$
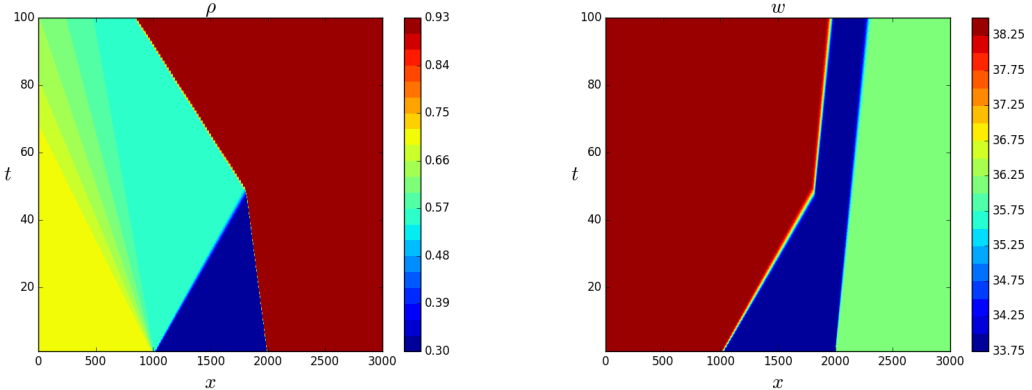

Figure 12. The contour plot for the density $\rho$ and for the maximal speed $w$ obtained with the Godunov method, with the parameters (12) and (19), and with $\psi(\rho)=1-\rho$. The initial condition are in (18). A shock curve is generated at about $x \sim 1800$ and interacts with a rarefaction curve generated at about $x \sim 2500$. 


\section{The Godunov method for a 2-phase model}

In Figure 13 the solution of the Cauchy problem, obtained with a wavefront tracking method, is drawn. Note that the structure of the numerical solution is essentially the same of the exact solution.
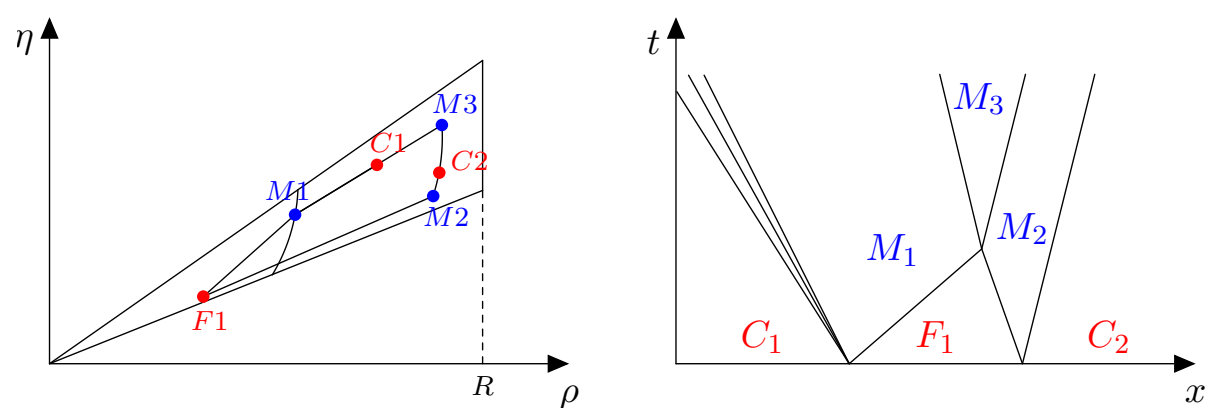

Figure 13. The solution to the Cauchy problem of Subsection 4.4, where $F_{1}=$ $(0.3,33.88), C_{1}=(0.7,38.33), C_{2}=(0.92,36.11)$. Note that $F_{1} \in F$, while $C_{1}, C_{2} \in C$. At left, in the $(\rho, \eta)$ plane, the initial states $F_{1}, C_{1}$, and $C_{2}$, and the states $M_{1}, M_{2}$, and $M_{3}$ obtained by solving the various Riemann problems. At right, the solution in the $(t, x)$ plane. The solution to the Riemann problem with initial conditions $\left(C_{1}, F_{1}\right)$ is given by a rarefaction wave, connecting $C_{1}$ to the intermediate state $M_{1}$, and by a wave of the second family. The solution to the Riemann problem with initial conditions $\left(F_{1}, C_{2}\right)$ is given by a shock wave, connecting $F_{1}$ to the intermediate state $M_{2}$, and by a wave of the second family. The solution to the Riemann problem with initial conditions $\left(M_{1}, M_{2}\right)$ is given by a shock wave, connecting $M_{1}$ to the intermediate state $M_{3}$, and by a wave of the second family.

\section{Acknowledgments}

The authors were partial supported by the INdAM-GNAMPA 2016 project "Balance Laws: Theory and Applications".

\section{REFERENCES}

1. R. M. Colombo, F. Marcellini, and M. Rascle, A 2-phase traffic model based on a speed bound, SIAM J. Appl. Math., vol. 70, no. 7, pp. 26522666,2010

2. S. Blandin, D. Work, P. Goatin, B. Piccoli, and A. Bayen, A general phase transition model for vehicular traffic, SIAM J. Appl. Math., vol. 71, no. 1, pp. 107-127, 2011.

3. M. J. Lighthill and G. B. Whitham, On kinematic waves. II. A theory of traffic flow on long crowded roads, Proc. Roy. Soc. London. Ser. A., vol. 229, pp. 317-345, 1955.

4. P. I. Richards, Shock waves on the highway, Operations Res., vol. 4, pp. 42-51, 1956. 


\section{Garavello, F. Marcellini}

5. B. S. Kerner, The physics of traffic: empirical freeway pattern features, engineering applications, and theory. Springer, 2012.

6. R. M. Colombo, Hyperbolic phase transitions in traffic flow., SIAM J. Appl. Math., vol. 63, no. 2, pp. 708-721, 2002.

7. R. M. Colombo, Phase transitions in hyperbolic conservation laws, in Progress in analysis, Vol. I, II (Berlin, 2001), pp. 1279-1287, World Sci. Publ., River Edge, NJ, 2003.

8. R. M. Colombo, P. Goatin, and F. S. Priuli, Global well posedness of traffic flow models with phase transitions, Nonlinear Anal., vol. 66, no. 11, pp. 2413-2426, 2007.

9. P. Goatin, The Aw-Rascle vehicular traffic flow model with phase transitions, Math. Comput. Modelling, vol. 44, no. 3-4, pp. 287-303, 2006.

10. J. P. Lebacque, X. Louis, S. Mammar, B. Schnetzlera, and H. HajSalem, Modélisation du trafic autoroutier au second ordre, Comptes Rendus Mathematique, vol. 346, pp. 1203-1206, November 2008.

11. F. Marcellini, Free-congested and micro-macro descriptions of traffic flow, Discrete Contin. Dyn. Syst. Ser. S, vol. 7, no. 3, pp. 543-556, 2014.

12. S. K. Godunov, A difference method for numerical calculation of discontinuous solutions of the equations of hydrodynamics, Mat. Sb. (N.S.), vol. 47 (89), pp. 271-306, 1959.

13. R. J. LeVeque, Finite volume methods for hyperbolic problems. Cambridge Texts in Applied Mathematics, Cambridge University Press, Cambridge, 2002.

14. C. Chalons and P. Goatin, Godunov scheme and sampling technique for computing phase transitions in traffic flow modeling, Interfaces Free Bound., vol. 10, no. 2, pp. 197-221, 2008.

15. X. Zhong, T. Y. Hou, and P. G. LeFloch, Computational methods for propagating phase boundaries, J. Comput. Phys., vol. 124, no. 1, pp. 192-216, 1996.

16. R. J. LeVeque, Numerical methods for conservation laws. Lectures in Mathematics ETH Zürich, Basel: Birkhäuser Verlag, second ed., 1992.

17. R. Courant, K. Friedrichs, and H. Lewy, On the partial difference equations of mathematical physics, IBM J. Res. Develop., vol. 11, pp. 215234, 1967. 